\title{
Local scale invariance as dynamical space-time symmetry in phase-ordering kinetics
}

\author{
Malte Henkel $^{a}$ and Michel Pleimling ${ }^{b}$ \\ ${ }^{a}$ Laboratoire de Physique des Matériaux, ${ }^{*}$ Université Henri Poincaré Nancy I, B.P. 239, \\ F - 54506 Vandouvre lès Nancy Cedex, France \\ ${ }^{b}$ Institut für Theoretische Physik I, Universität Erlangen-Nürnberg, D - 91058 Erlangen, Germany
}

\begin{abstract}
The scaling of the spatio-temporal response of coarsening systems is studied through simulations of the $2 D$ and $3 D$ Ising model with Glauber dynamics. The scaling functions agree with the prediction of local scale invariance, extending dynamical scaling to a space-time dynamical symmetry.
\end{abstract}

05.20-y, 64.60.Ht, 75.40.Gb, 11.25.Hf

Ageing phenomena are observed in a broad variety of systems with slow relaxation dynamics. Useful insight may often be gained via the consideration of simple ferromagnetic models (rather than genuinely glassy systems), quenched to a temperature $T<T_{c}$ from a disordered initial state into the low-temperature phase. For their study two-time quantities, rather than the more usual one-time quantities, are particularly useful, see [1-3] for recent reviews. Those display dynamical self-similarity in the ageing regime where the order-parameter autocorrelator $C(t, s)=\langle\phi(t) \phi(s)\rangle$ decays from its plateau value $q_{\mathrm{EA}}=M_{\mathrm{eq}}^{2}$ to zero $\left(M_{\mathrm{eq}}\right.$ is the spontaneous magnetization). In this regime both $s$ and $\tau=t-s$ are much larger than the microscopic time scale (set to unity). Similarly, the autoresponse function, defined as $R(t, s)=\delta\langle\phi(t)\rangle / \delta h(s)$ with $t>s$ shows a scaling behaviour such that the scaling laws

$$
C(t, s) \simeq M_{\mathrm{eq}}^{2} f_{C}(t / s), R(t, s) \simeq s^{-1-a} f_{R}(t / s)
$$

with $f_{C}(1)=1$ are found to hold for a broad range of models [1-3]. For $x \gg 1$, the scaling functions usually fall off as $f_{C}(x) \sim x^{-\lambda_{C} / z}$ and $f_{R}(x) \sim x^{-\lambda_{R} / z}$, where $z$ is the dynamic critical exponent, $\lambda_{C}$ is the autocorrelation exponent $[4,5]$ and $\lambda_{R}$ is the autoresponse exponent $[6]$. We shall focus here on phase-ordering in the Glauber-Ising model in $d>1$ dimensions where $a=1 / z=1 / 2$ is expected $[7,2]$. Recent arguments [8] leading to $a=1 / 4$ in the $2 D$ Glauber-Ising model have been rejected through a detailed study of the scaling of $R(t, s)$, the results of which reconfirm $a=1 / 2[9]$.

Eq. (1) states that the two-time quantities evaluated at the same spatial location transform covariantly under a global rescaling of time $t \rightarrow b t$ with $b$ constant. Recently, it has been proposed that the response functions should transform covariantly under local scale transformations with $b=b(t)$, but with time-translations excluded [10]. By analogy with conformal invariance, in particular covariance of $R$ under the so-called 'special' transformations which transform time as $t \rightarrow t /(1+\gamma t)$, is assumed. If that is the case, the exact autoresponse scaling function becomes $[10,11]$

$$
f_{R}(x)=r_{0} x^{1+a-\lambda_{R} / z}(x-1)^{-1-a}
$$

and depends on the universality classes only through the values of the exponents $a$ and $\lambda_{R} / z$, while $r_{0}$ merely is a normalization constant. This prediction of local scale invariance (LSI) has been confirmed in a variety of physically very different models, see [10] and references therein. Still, better evidence in favour of LSI than mere phenomenology would be desirable.

The origin of LSI can be understood in the special case $z=2$ (which is realized in all cases of phase-ordering kinetics we are going to consider [12]). A Ward identity can be written down such that if the system is known to be (i) scale-invariant with $z=2$ and (ii) Galilei-invariant, then its invariance under the full group of local scale transformations follows [13]. Therefore, scale invariance and Galilei invariance appear as the building blocks for LSI. So far, no direct test of Galilei invariance in phaseordering systems was ever performed.

Direct tests of Galilei invariance require the study of the time- and space-dependence of response functions. For phase-ordering, it is natural to expect a scaling behaviour of the linear response function

$$
R(t, s ; \mathbf{r})=\left.\frac{\delta\left\langle\phi_{\mathbf{r}}(t)\right\rangle}{\delta h_{\mathbf{0}}(s)}\right|_{h=0}=s^{-1-a} F_{R}\left(\frac{t}{s}, \frac{\mathbf{r}}{(t-s)^{1 / z}}\right)
$$

with the scaling function $F_{R}(x, \mathbf{u})$ and $F_{R}(x, \mathbf{0})=f_{R}(x)$. For $z=2$, Galilei invariance (combined with the Ward identity of LSI) predicts $[14,10,13]$

$$
R(t, s ; \mathbf{r})=R(t, s) \exp \left(-\frac{\mathcal{M}}{2} \frac{\mathbf{r}^{2}}{t-s}\right)
$$

where $R(t, s)$ is the autoresponse function given by eqs. $(1,2)$ and $\mathcal{M}$ is a direction-dependent non-universal constant. Eq. (3) gives the full spatio-temporal scaling of the linear response. We shall sucessfully test it in the $2 D$ and $3 D$ kinetic Glauber-Ising models and provide thereby the first direct evidence of Galilei invariance in a phaseordering system. Since the $2 D / 3 D$ Glauber-Ising model cannot be reduced to a free-field theory, it is highly nontrivial that its exact response function takes the simple free-field form eq. (3).

As precise simulational data for the autoresponse $R(t, s)$ are difficult to obtain, it is convenient to study instead the thermoremanent magnetization [15] 


$$
T M_{\mathrm{TRM}}(t, s) / h_{(0)}=\rho(t, s)=T \int_{0}^{s} \mathrm{~d} u R(t, u)
$$

We consider the Ising model on a hypercubic lattice, with periodic boundary conditions and the Hamiltonian $\mathcal{H}=-\sum_{(\mathbf{i}, \mathbf{j})} \sigma_{\mathbf{i}} \sigma_{\mathbf{j}}$. We use heat-bath dynamics defined through the stochastic rule

$\sigma_{\mathbf{i}}(t+1)= \pm 1$ with probability $\frac{1}{2}\left[1 \pm \tanh \left(h_{\mathbf{i}}(t) / T\right)\right]$

with the local field $h_{\mathbf{i}}(t)=\sum_{\mathbf{a}(\mathbf{i})} \sigma_{\mathbf{a}}(t)$ and where $\mathbf{a}(\mathbf{i})$ runs over the nearest neighbours of the site i. $M_{\mathrm{TRM}}(t, s)$ is measured by applying a quenched spatially random magnetic field $\pm h_{(0)}$ for times between the quench at $t=0$ and the waiting time $s$ [15]. The presence of this external field then changes the local field in eq. (5) to $h_{\mathbf{i}}(t)=\sum_{\mathbf{a}(\mathbf{i})} \sigma_{\mathbf{a}}(t) \pm h_{(0)}$.

It has been understood recently that the scaling behaviour of $M_{\mathrm{TRM}}$ for $s \gg 1$ is not a simple power law but rather shows a cross-over behaviour $[16,8]$. It is of the form [9]

$$
\rho(t, s)=r_{0} s^{-a} f_{M}(t / s)+r_{1} s^{-\lambda_{R} / z} g_{M}(t / s)
$$

provided the system was initially prepared at infinite temperature. Here the correction term can be estimated as $g_{M}(x) \simeq x^{-\lambda_{R} / z}$, while the scaling function $f_{M}(x)$ can be found from local scale invariance using eqs. $(1,2,4)$ and is given explicitly by eq. (5.47) in [10]. Finally, $r_{0,1}$ are normalization constants. In practice, it turns out that $\lambda_{R}$ is quite close to its lower bound $d / 2$ and in particular in $2 D$, the correction to the leading scaling behaviour is sizeable. Before any meaningful study of the spatiotemporal response can be carried out, the correction term must be subtracted off.

As explained in [9], this can be carried out by fixing a value of $x=t / s$. Then $r_{0,1}$ are obtained by fitting eq. (6) to the computed thermoremanent magnetization $M_{\text {TRM }}$. In order to illustrate the quality of this procedure, we show in figure 1 data for the scaling function $f_{M}(x)$ obtained after subtraction of the correction term $r_{1} s^{-\lambda_{R} / z} g_{M}(t / s)$ from the integrated autoresponse $\rho$ of the $2 D$ and $3 D$ Glauber-Ising model. Statistical error bars in $2 D$ are smaller than the symbol size and in $3 D$ are of the order of the scatter in the data. In table I, we list the values of $\lambda_{R}[4,17]$ and the constants $r_{0,1}$ which were determined at $x=7$. We find a nice data collapse and a clear agreement with the LSI prediction.

Having thus checked the expected scaling of the autoresponse function, we can now turn towards the spatiotemporal response. Again, we consider the integrated response rather than $R(t, s ; \mathbf{r})$ because it is considerably less affected by noise. In order to fix the non-universal parameter $\mathcal{M}$ in (3), we form

$$
\rho_{0}(t, s ; \mathbf{r})=T \int_{0}^{s} \mathrm{~d} \tau R(t, s-\tau ; \mathbf{r})
$$

which is measured on the lattice by keeping a small random magnetic field $\pm h_{(0)}$ until the waiting time $s$ at the site $\mathbf{0}$ and then observing the thermoremanent magnetization $M_{\mathrm{TRM}}(t, s ; \mathbf{r})=h_{(0)} \rho_{0}(t, s ; \mathbf{r}) / T=\overline{\left\langle h_{\mathbf{0}} \sigma_{\mathbf{r}}(t)\right\rangle} / T$ at a different site $\mathbf{r}$ (where $\langle\cdots\rangle$ indicates an average over the thermal noise whereas the bar means an average over the random field distribution). We computed the spatially and temporally integrated response for the Ising model with Glauber dynamics in two and three dimensions. The two-dimensional systems usually contained $300^{2}$ spins on a square lattice, whereas in three dimensions cubic lattices with typically $60^{3}$ sites were considered. Some Monte Carlo simulations were also done for other system sizes in order to check against finite-size effects. Since $\rho_{0}(t, s ; \mathbf{r})$ is very noisy, one must average over a large number of runs with different realizations of the thermal noise and of the spatially random magnetic field. For every waiting time we avaraged over at least 50000 different runs. The whole study took about $2 \cdot 10^{5}$ CPU hours on a SGI Origin 3800 parallel computer.

Following the same lines as in [9], we arrive at the scaling form [18], where $\mathbf{r}$ varies along a fixed direction on the lattice

$$
\begin{aligned}
\rho_{0}(t, s ; \mathbf{r}) \simeq & \rho_{\infty}(t-s ; \mathbf{r})+r_{0} s^{-a} F_{0}\left(\frac{t}{s}, \mathcal{M} \frac{r^{2}}{s}\right) \\
& +r_{1} s^{-\lambda_{R} / z} G_{0}\left(\frac{t}{s}, \mathcal{M} \frac{r^{2}}{s}\right)
\end{aligned}
$$

where the universal scaling functions read

$$
\begin{aligned}
& F_{0}(x, y)=\int_{0}^{1} \mathrm{~d} v \exp [-y / 2(x-1+v)] h_{R}(x, v) \\
& G_{0}(x, y) \simeq x^{-\lambda_{R} / z} e^{-y / 2 x}
\end{aligned}
$$

with $h_{R}(x, v):=f_{R}\left(\frac{x}{1-v}\right)(1-v)^{-1-a}$ where $\mathcal{M}$ is a direction-dependent new parameter. In this work we choose $T$ such as to keep anisotropy effects small and also to avoid the cross-over to critical dynamics at $T=T_{c}$. This is illustrated in figure 2 , for the $2 D$ case at $T=1.5$ where we plot $F_{0}$ as function of $r^{2} / s$. For the displayed data points $r$ varies between 1 and $\sqrt{s}$ when going along the (10) direction and between $\sqrt{2}$ and $\sqrt{2 s}$ when going along the (11) direction. At the considered temperature any anisotropies are very small and the values of $\mathcal{M}$, determined separately in the two directions, coincide, $\mathcal{M}_{(10)}=\mathcal{M}_{(11)}=\mathcal{M}$. Similar results also hold in $3 D$ at $T=3$. We fix $\mathcal{M}$ at $x=7$ (see table I and inset) using (8) and then obtain a parameter-free prediction for other values of $x$. This is shown in the inset of figure 2 , where the LSI-prediction is compared to numerical data for two additional values of $x$ and two different waiting times. Since by now all non-universal parameters are fixed, this constitutes by itself a quantitative test of local scale invariance. 
A fuller test of the spatio-temporal response is obtained by considering the spatially and temporally integrated response function

$$
\frac{\mathrm{d} \rho(t, s ; \mu)}{\mathrm{d} \Omega}=T \int_{0}^{s} \mathrm{~d} u \int_{0}^{\sqrt{\mu s}} \mathrm{~d} r r^{d-1} R(t, u ; \mathbf{r})
$$

where the space integral is along a straight line of length $\Lambda=\sqrt{\mu s}$ but we do allow for the possibility of an anisotropy as a function of the solid angle $\Omega$. Such anisotropies are known to occur if $T<T_{c}$ [19]. As before, we derive from (3) the scaling form [18]

$$
\begin{aligned}
& \frac{\mathrm{d} \rho(t, s ; \mu)}{\mathrm{d} \Omega}=\rho^{(1)}\left(t-s, \mathbf{r}^{2}\right) \\
& +r_{0} s^{d / 2-a} \rho^{(2)}(t / s, \mu)+r_{1} s^{d / 2-\lambda_{R} / z} \rho^{(3)}(t / s, \mu)
\end{aligned}
$$

with the explicit scaling functions

$$
\begin{aligned}
\rho^{(2)}(x, \mu) & =\frac{\mu^{d / 2}}{d} \int_{0}^{1} \mathrm{~d} v h_{R}(x, v) \mathcal{F}_{d}\left(\frac{\mathcal{M} \mu}{x-1+v}\right) \\
\rho^{(3)}(x, \mu) & \simeq \frac{\mu^{d / 2}}{d} x^{-\lambda_{R} / z} \mathcal{F}_{d}\left(\frac{\mathcal{M} \mu}{x}\right) \\
\mathcal{F}_{d}(y) & =e^{-y / 2}{ }_{1} \mathrm{~F}_{1}\left(1,1+\frac{d}{2} ; \frac{y}{2}\right)
\end{aligned}
$$

This is the general expression for the scaling of the spatiotemporally integrated response function. If we fix $\mu$ and let $x=t / s$ vary, the form of the scaling function of $s^{a-d / 2} \mathrm{~d} \rho / \mathrm{d} \Omega$ merely depends on $\mu$. We stress that the exponent $\lambda_{R}$ and the free parameters $r_{0}, r_{1}, \mathcal{M}$ are now all fixed such that there remains no free fitting parameter at all when comparing (11) with our numerical data.

It is of interest to compare the maximal distance $r_{\max }$ accessed by our simulation with the physical length scale of the problem, for example the domain size $L(s)$ at the instant the magnetic field is turned off. From the correlation function, in $2 D$ we estimate $L(s) \approx 3,6,9.5$ for $s=25,100,225$, respectively, with $h_{(0)}=0.05$. In $3 D$, we find $L(s) \approx 2.1,3.3,4$ for $s=25,64,100$. As expected $L(s) \sim s^{1 / z}$, with $z \approx 2$. The value $\mu=4(\mu=2)$ corresponds to $r_{\max } / L(s) \approx 3.2(3.4)$ in $2 D(3 D)$, respectively and we see that the data probe the large-distance region, well beyond the domain size $L(s)$.

The integrated spatio-temporal response in $2 D$ at $T=$ 1.5 is shown in figure 3 for two values of $\mu$ and where the correction $\rho^{(3)}$ was already subtracted off. We find a nice scaling behaviour over the whole range of waiting times we could consider. We stress that the agreement between local scale invariance (3) and our data for several values of $\mu$ is a real test, since no free parameter remains. In particular, both the height and the position of the maximum of the scaling function for $\mu=2$ and 4 is completely fixed. We can conclude that LSI is fully vindicated. This is the first time that the exact functional form of the scaling function of the spatio-temporal response of a generic non-equilibrium spin system is found.
Similarly, the integrated spatio-temporal response in $3 D$ at $T=3$ is shown in figure 4 . Again, a nice scaling behaviour in perfect agreement with LSI is found.

The prediction (3) is also verified in a few exactly solvable models with a non-conserved order parameter and undergoing phase-ordering kinetics with $z=2$. In particular, (3) holds in the $d$-dimensional kinetic spherical model with $d>2$ quenched to either $T<T_{c}$ or $T=T_{c}$ and independently of initial correlations $[20-22,16,6]$.

Summarizing, we presented the first quantitative study of the scaling of the spatio-temporal response in coarsening systems. For the first time, we find direct evidence for Galilei invariance in the ageing regime of a phaseordering system. This is a new dynamic symmetry whose presence in a non-equilibrium phase transition was not anticipated. Our result provides a strong indication that LSI is indeed a true space-time dynamical symmetry of statistical systems undergoing phase-ordering kinetics.

We thank B.P. Vollmayr-Lee and C. Godrèche for discussions. This work was supported by the BayerischFranzösisches Hochschulzentrum (BFHZ) and by CINES Montpellier (projet pmn2095).

* Laboratoire associé au CNRS (UMR 7556)

[1] J.P. Bouchaud in M.E. Cates and M.R. Evans (eds) Soft and fragile matter, IOP (Bristol 2000)

[2] C. Godrèche and J.-M. Luck, J. Phys. Cond. Matt. 14, 1589 (2002) and references therein.

[3] L.F. Cugliandolo, cond-mat/0210312

[4] D.S. Fisher and D.A. Huse, Phys. Rev. B38, 373 (1988).

[5] D.A. Huse, Phys. Rev. B40, 304 (1989).

[6] A. Picone and M. Henkel, J. Phys. A35, 5575 (2002).

[7] L. Berthier, J.L. Barrat, and J. Kurchan, Eur. Phys. J. B11, 635 (1999).

[8] F. Corberi, E. Lippiello and M. Zannetti, Eur. Phys. J. B24, 359 (2001); Phys. Rev. E65, 046136 (2002); Phys. Rev. Lett. 90, 099601 (2003).

[9] M. Henkel, M. Paeßens and M. Pleimling, Europhys. Lett. 62, 664 (2003); M. Henkel and M. Pleimling, Phys. Rev. Lett. 90, 099602 (2003).

[10] M. Henkel, Nucl. Phys. B641, 405 (2002).

[11] M. Henkel, M. Pleimling, C. Godrèche, and J.-M. Luck, Phys. Rev. Lett. 87, 265701 (2001).

[12] For $z=2$ the LSI-group is the Schrödinger group, the max. kinematic group of the free Schrödinger equation.

[13] M. Henkel and J. Unterberger, Nucl. Phys. B660, 407 (2003)

[14] M. Henkel, J. Stat. Phys. 75, 1023 (1994).

[15] A. Barrat, Phys. Rev. E57, 3629 (1998).

[16] W. Zippold, R. Kühn, and H. Horner, Eur. Phys. J. B13, 531 (2000).

[17] K. Humayun and A.J. Bray, J. Phys. A24, 1915 (1991).

[18] the quasiequilibrium terms $\rho_{\infty}, \rho^{(1)}$ in $(8,11)$ vanish.

[19] A.D. Rutenberg, Phys. Rev. E54, R2181 (1996); A.D. Rutenberg and B.P. Vollmayr-Lee, Phys. Rev. Lett. 83, 3772 (1999).

[20] T.J. Newman and A.J. Bray, J. Phys. A23, 4491 (1990).

[21] H.K. Janssen, B. Schaub, and B. Schmittmann, Z. Phys. B73, 539 (1989). 
[22] C. Godrèche and J.M. Luck, J. Phys. A33, 9141 (2000).
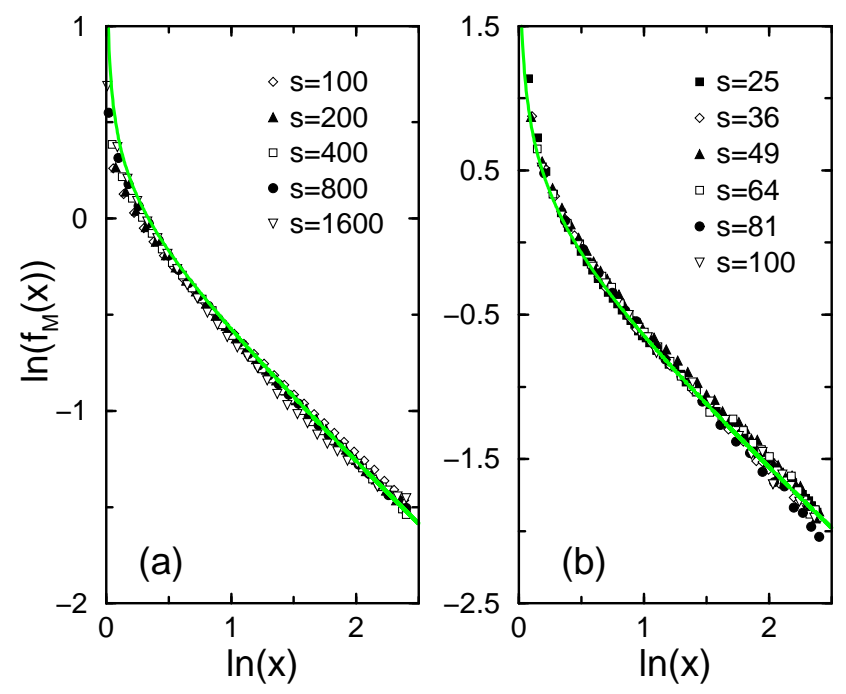

FIG. 1. Scaling of the integrated response function $\rho$ for the Glauber-Ising model. (a) $2 D$ at temperature $T=1.5$ (b) $3 D$ at $T=3$. The symbols correspond to different waiting times. The full curves are obtained by integrating (2).

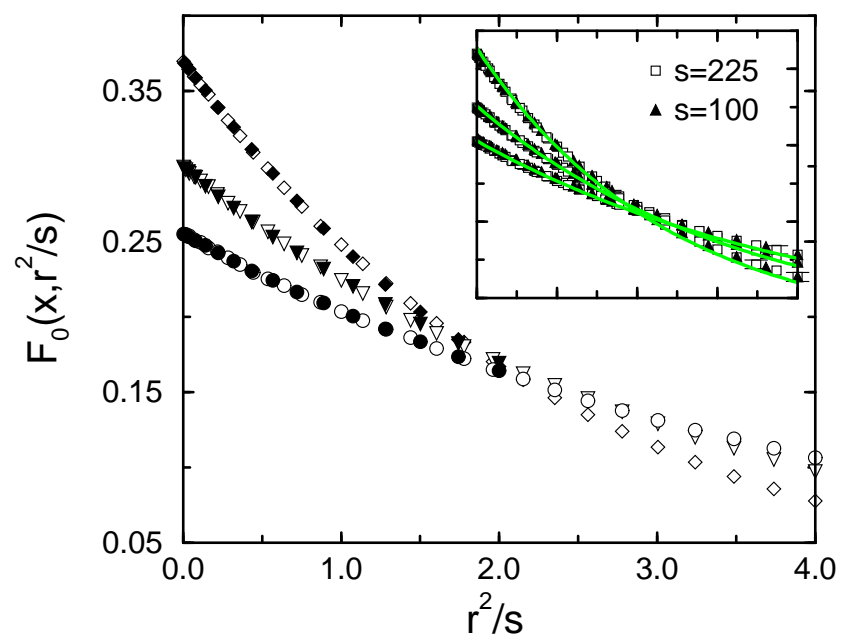

FIG. 2. Scaling of the temporally integrated response function $\rho_{0}$ for the $2 D$ Glauber-Ising model at $T=1.5$ and $s=225$. Data obtained along the (10) resp. (11) direction are shown by open resp. filled symbols. Diamonds: $x=5$, triangles: $x=7$, circles: $x=9$. Inset: Determination of the mass $\mathcal{M}$, see main text. Some typical error bars are shown in the inset.
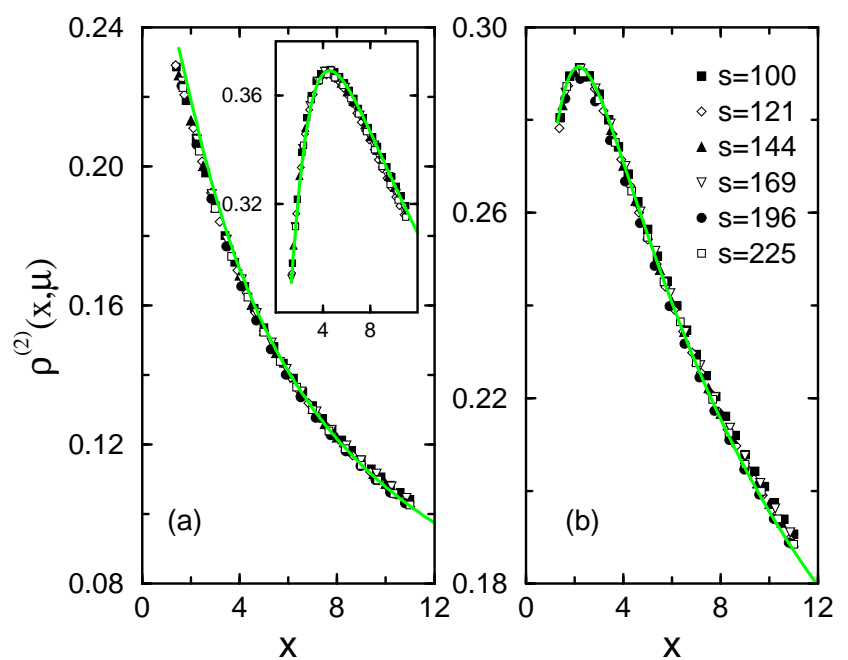

FIG. 3. Scaling of the integrated spatio-temporal response function $\rho$ for the $2 D$ Glauber-Ising model at $T=1.5$ and at (a) $\mu=1$ (inset: $\mu=4$ ) and (b) $\mu=2$ (integrating (a) along the (10) direction and (b) along the (11) direction). The full curves are from eq. (12). Error bars are smaller than the symbol size.
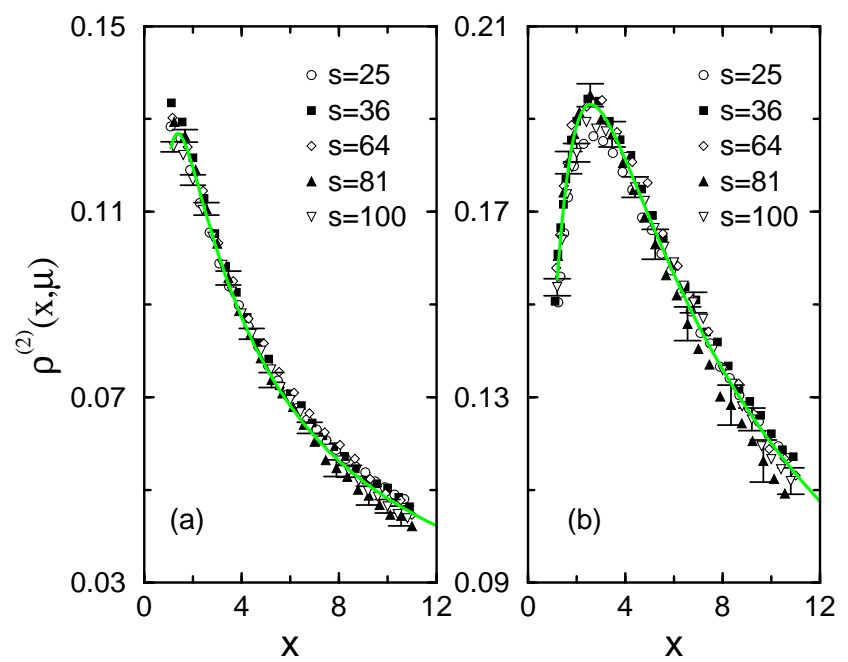

FIG. 4. Scaling of the integrated spatio-temporal response function $\rho$ for the $3 D$ Glauber-Ising model at $T=3$ and at (a) $\mu=1$ and (b) $\mu=2$ (integrating (a) along the (100) direction and (b) along the (110) direction). The full curves are from eq. (12). Some typical error bars are also shown.

TABLE I. Values of the autoresponse exponent $\lambda_{R}$ and of the parameters $r_{0}, r_{1}$ and $\mathcal{M}$ in the Glauber-Ising model in two dimensions at $T=1.5$ and in three dimensions at $T=3$.

\begin{tabular}{r|rrrr|}
\hline \hline & $\lambda_{R}$ & \multicolumn{1}{c}{$r_{0}$} & \multicolumn{1}{c}{$r_{1}$} & $\mathcal{M}$ \\
\hline $2 D$ & 1.26 & $2.65 \pm 0.05$ & $-2.76 \pm 0.05$ & $4.08 \pm 0.04$ \\
$3 D$ & 1.60 & $0.31 \pm 0.02$ & $0.61 \pm 0.02$ & $4.22 \pm 0.05$ \\
\hline \hline
\end{tabular}

